\title{
Dynamics of impairment during and after treatment: the AMFES cohort
}

\author{
A. MEIMA ${ }^{+}$, P. R. SAUNDERSON*, S. GEBRE*, \\ K. DESTA* \& J. D. F. HABBEMA ${ }^{+}$ \\ ${ }^{+}$Department of Public Health, Erasmus University Rotterdam, \\ The Netherlands \\ *All Africa Leprosy, Tuberculosis and Rehabilitation Training Centre, \\ Addis Ababa, Ethiopia
}

\section{Accepted for publication 1 May 2001}

Summary This study investigates the dynamics of impairment during and after multidrug therapy treatment for the patient cohort of the prospective ALERT MDT Field Evaluation Study (AMFES). The impairment status was compared at intake, at release from treatment ( $\mathrm{rt}$ ), and at the time of the latest survey between 24 and 48 months after release from treatment (follow-up). The eye-hand-foot impairment score (EHF score), which is the sum of the WHO impairment grades of the eyes, hands, and feet, was used as tool for comparison. In all, 433 out of the 592 patients (224 PB and $209 \mathrm{MB})$ completed treatment in time and were assessed at release from treatment. The risk of getting impaired was $4 \%$ for the $113 \mathrm{~PB}$ and $21 \%$ for the $91 \mathrm{MB}$ patients who were initially free from impairment. Out of the 111 initially impaired PB patients, $41 \%$ recovered or improved and $13 \%$ worsened in EHF score. For the 118 initially impaired MB patients, these figures were: recovery or improvement $43 \%$ and worsening $13 \%$. Three hundred and twenty-three out of the 433 patients (158 PB and $165 \mathrm{MB}) \mathrm{had}$ a follow-up examination in between the next 24-48 months after rft. The risks of impairment at follow-up were $6 \%$ for the 79 PB and $18 \%$ for the $77 \mathrm{MB}$ patients without impairment at rft. Out of the $79 \mathrm{~PB}$ patients with impairment at $\mathrm{rft}$, $35 \%$ recovered or improved and $28 \%$ worsened. For the 88 impaired MB patients, these figures were: recovery or improvement $26 \%$ and worsening $27 \%$. Patients showed a tendency to compensate EHF score improvement before rft by worsening after $\mathrm{rft}$ and vice versa. The first main conclusion is that the impairment status at intake was by far the most important determinant for future impairment. The second one is that the dynamics of impairment were less favourable after rft than before. Little is known about the long-term fate of leprosy patients with irreversible nerve damage and the associated risk of developing severe secondary impairment. Especially in this era of the leprosy elimination goal, we should give this accumulating patient group due attention in research and health policy agendas.

Correspondence to: A. Meima, Department of Public Health, Faculty of Medicine and Health Sciences, Erasmus University Rotterdam, PO Box 1738, 3000 DR Rotterdam, The Netherlands (e-mail: Meima@mgz.fgg.eur.nl) 


\section{Introduction}

Newly detected leprosy patients may or may not present with impairments. During and after multidrug therapy (MDT), new impairments may develop, and existing impairments may worsen, remain stable or improve. Many studies have addressed the presence of impairments in newly detected patients. ${ }^{1-7}$ Less attention has been paid to the dynamics of impairment during and especially after MDT treatment. One study addressed patients in Malawi; ${ }^{8}$ other studies were conducted in Asian countries. ${ }^{3,5,9,10}$ The percentage of newly detected patients presenting with impairment varied considerably across these studies. Whereas worsening of impairment status was not negligible, the studies revealed that a majority of patients with impairment at release from MDT already had impairment at registration. In only one of these studies, ${ }^{5}$ the impairment dynamics were evaluated during a follow-up period that extended well beyond release from treatment.

All studies employed WHO disability grades. The use of the term 'disability' is however questionable. According to the International Classification of Impairments, Disabilities and Handicaps (ICIDH), ${ }^{11}$ disability refers to inability to perform activities due to impairment. Impairment is defined as 'any loss or abnormality of psychological, physiological, or anatomical structure or function'. Under ICIDH, the WHO grades do not reflect disability but impairment. ${ }^{10}$ Following earlier publications, ${ }^{7,9,10}$ this paper will therefore use the term 'WHO impairment grade' instead of 'WHO disability grade'. In the generally known 1988 WHO system, ${ }^{12}$ grades are assigned to each eye, hand and foot using a scale with three possible outcomes $(0,1,2)$. The maximum of these six grades, the 'maximum WHO impairment grade', specifies the patient's overall score. The 1988 WHO system has again been updated in 1998 by re-defining the grades for the eyes. $^{13}$

The maximum WHO impairment grade recognizes both first onset of impairment and total recovery of existing impairment. Otherwise, its sensitivity to improvement or worsening of impairments is limited. This is of concern because from patient registration onwards, the performance of the services of a leprosy control programme is expressed in changes in impairment and disability. Accordingly, the 1988 maximum WHO impairment grade has primarily been applied to compare impairment profiles of newly detected patients across countries. ${ }^{14}$ De Rijk et al. introduced an alternative summary score, ${ }^{15}$ which uses the sum instead of the maximum of the individual grades for eyes, hands and feet. Further study ${ }^{10}$ of impairment dynamics promoted the so-called Eye-HandFoot impairment score (EHF score), as demonstrating a higher sensitivity in registering change than the maximum WHO grade. The EHF score has also been suggested as tool for evaluating the effectiveness of steroid programmes. ${ }^{16}$ The EHF score and the maximum WHO impairment grade share the advantage that their components - the individual grades for eyes, hands and feet - are routinely recorded in leprosy control programmes.

In this paper, the EHF score is applied to investigate the severity and evolution of impairment over time for the cohort of the ALERT MDT Field Evaluation Study (AMFES). Comparisons are made between EHF scores at intake, at release from treatment, and at the latest survey examination between 24 and 48 months after release from treatment. Objectives and interim results of the AMFES study, which is conducted within a routine leprosy control programme in central Ethiopia, were described before by de Rijk et al. ${ }^{15,17}$ 


\section{Materials and methods}

Methods of enrolment, diagnosis, administration of fixed-duration MDT and case holding in AMFES have previously been specified ${ }^{15}$ and reviewed ${ }^{18}$. The present study involves all enrolled newly detected patients except those who had errors in enrolment procedures or in diagnosis.

Recorded patient characteristics include age, sex, clinical classification and bacteriological index (BI). The type of treatment (PB or $\mathrm{MB}$ ) was chosen on the basis of clinical classification and skin smears. For clinical classification, the simplified system for field workers recommended by Jopling ${ }^{19}$ was used. It should be recognized that many patients correctly diagnosed as PB in the present study would be classified as MB if presently used criteria focussing on number of skin lesions or number of body areas affected had been applied. $^{13}$

AMFES patients were scheduled for examination at intake, while on MDT, at release from treatment, at 3 and 6 months after release from treatment (rft) and thereafter at intervals of 6 months. Examination involved the recording of the WHO impairment grades for the eyes, hands and feet according to the 1988 WHO grade definitions. ${ }^{12}$ The maximum WHO impairment grade and the sum of these six impairment grades for the eyes, hands and feet EHF score (ranging from 0 to 12 ) - follow directly. This paper investigates the dynamics of impairment by comparing the EHF scores at three different points in time: intake, release from treatment, and the time of the latest survey conducted between 24 and 48 months after release from treatment. A considerable number of patients did not complete treatment, and some patients did complete treatment in due time but were not examined at release from treatment. Patients who did complete treatment and who were examined at release from treatment are referred to as 'rft patients'. Those among the 'rft patients' who in addition had a survey examination between 24 and 48 months after release from treatment are in this study denoted as 'follow-up' patients. Unless indicated otherwise, the term 'worsening' will refer to any increase in EHF score (this includes onset of impairment in previously unimpaired patients). 'Improvement' refers to a decrease in EHF score, while 'recovery' indicates that the EHF score has decreased to zero from a previously positive score.

In the data analysis, statistical significance refers to the 5\% level. Frequency distributions were compared using the Chi-square test. The data analysis was carried out in SPSS.

\section{Results}

A total of 603 new patients were enrolled in the AMFES project. Out of these, 11 patients were excluded from the present data analysis because of either incorrect enrolment procedures or incorrect diagnosis. The resulting study cohort consists of 292 PB and 300 MB patients.

\section{THE COHORT OVER TIME}

Out of the 592 patients, 454 patients completed treatment in time. The treatment completion rates were higher for PB than for MB patients (PB: 242/292, or 83\%, against MB: 212/300, or $71 \%, P<0.001)$. Thirteen patients died before they could be released from treatment, $104 \mathrm{did}$ not complete treatment and were lost to follow-up, and 21 did not complete treatment in due 
time but were seen later. Twenty-one patients completed treatment in time but were not examined by the leprosy control supervisor (LCS) at the end of treatment. So, 433 (224 PB and $209 \mathrm{MB}$ ) patients completed treatment in time and were examined by the LCS at release from treatment.

The $433 \mathrm{rft}$ patients differed from the other 159 patients in the cohort in several respects. Summarizing, the rft group included more children (PB: $22 \%$ against $7 \%, P<0 \cdot 01$; MB: $11 \%$ against $5 \%, P=0 \cdot 1)$. As compared to the non-rft group, the MB rft patients more often had high $\mathrm{BI}$ values $(\mathrm{BI} \geq 3$; 75\% against 63\%, $P<0.05)$ and less of ten had EHF scores of 3 or more ( $28 \%$ against $41 \%, P<0.05)$. The percentage with EHF score 3 or more was $25 \%$ for both PB rft and non-rft patients.

Out of the $433 \mathrm{rft}$ patients, 323 patients had a follow-up examination between 24 and 48 months after $\mathrm{rft}(158 / 224 \mathrm{~PB}$, or $71 \%$, against $165 / 209 \mathrm{MB}$, or $79 \% ; P<0 \cdot 05)$. In comparing these 'follow-up' patients with the $110 \mathrm{rft}$ patients without follow-up (66 PB and $44 \mathrm{MB}$ ), again differences are observed. Females were under-represented in the follow-up group of PB rft patients (37\% against $52 \%, P=0.05)$. The age distributions of the $\mathrm{PB}$ rft patients with and without follow-up also differed significantly. In the follow-up group, children were overrepresented (25\% against $15 \%$ ) and young adults (ages 15-29 years) were under-represented (30\% against $52 \%$ ). In MB patients, the percentages of females were similar (31\% against $30 \%$ ), whereas children and young adults together were over-represented in the follow-up group (58\% against $41 \%, P=0.05$ ). The rft patients with follow-up did not differ significantly from the rft patients without follow-up in percentage with BI 3 or more (MB patients only), percentage with EHF-score 3 or more (PB: $27 \%$ against 20\%, MB: $28 \%$ against 25\%) and percentages improving (including recovery) and worsening in EHF score between intake and $\mathrm{rft}$.

IMPAIRMENT AT INTAKE

Table 1 gives details of impairments at intake. About half of the patients had no impairment. Five percent of both $\mathrm{PB}$ and MB patients had just one extremity or eye affected with WHO grade 1 (EHF score 1). Thirty-three percent of PB and 39\% of MB patients had EHF scores ranging from 2 to 4 . The percentages with EHF score 5 or more were $11 \%$ for $\mathrm{PB}$ and $12 \%$ for $\mathrm{MB}$. Between $\mathrm{PB}$ and $\mathrm{MB}$, no significant differences were observed in percentage with any impairment (PB: 50\%, MB: 56\%) and with WHO grade 2 impairment (PB: 26\%, MB: 21\%). PB patients with EHF scores ranging from 2 to 4 more often had at least one extremity or eye affected with WHO grade 2 than MB patients (PB: $45 \%, \mathrm{MB}: 21 \%, P=0 \cdot 001$ ). All patients with EHF score 5 or more had at least one extremity or eye with grade 2 impairment. Further analysis showed that, with two exceptions for both PB and MB, they all had at least four extremities affected (for this analysis both eyes are included with the hands and feet to give a total of six 'extremities'). The large majority of the group of all impaired patients had at least two extremities affected (PB: 76\%, MB: 87\%). $5 / 224(2 \%)$ PB patients and 3/209 (1.4\%) MB patients had eye impairment: all these patients, except for one PB patient, had only grade 1 eye impairment. After re-examining patients with eye problems, data on eyes were corrected to refer to eye impairment that is due to leprosy only. Eye impairment figures earlier presented by de Rijk et al. ${ }^{15}$ were therefore higher. Overall, the distribution of EHF scores did not differ significantly between PB and MB patients. 
Table 1. Comparison between maximum WHO impairment grade and eye-hand-foot (EHF) score at intake for patients who completed treatment and who were assessed for impairment grades of eyes, hands and feet at release from treatment ('rft patients')

\begin{tabular}{|c|c|c|c|c|c|c|c|c|c|c|}
\hline \multirow{2}{*}{$\begin{array}{l}\text { Maximum } \\
\text { WHO grade }\end{array}$} & \multicolumn{8}{|c|}{ EHF score } & \multirow[b]{2}{*}{ Total } & \multirow[b]{2}{*}{ Percentage $(\%)$} \\
\hline & 0 & 1 & 2 & 3 & 4 & 5 & 6 & $7+$ & & \\
\hline \multicolumn{11}{|l|}{ PB patients } \\
\hline 0 & 113 & & & & & & & & 113 & 50 \\
\hline 1 & & 11 & 29 & 6 & 6 & & & & 52 & 23 \\
\hline 2 & & & 16 & 11 & 7 & 4 & 11 & 10 & 59 & 26 \\
\hline Total & 113 & 11 & 45 & 17 & 13 & 4 & 11 & 10 & 224 & \\
\hline Percentage (\%) & 50 & 5 & 20 & 8 & 6 & 2 & 5 & 4 & & 100 \\
\hline \multicolumn{11}{|l|}{ MB patients } \\
\hline 0 & 91 & & & & & & & & 91 & 44 \\
\hline 1 & & 10 & 45 & 11 & 9 & & & & 75 & 36 \\
\hline 2 & & & 5 & 5 & 7 & 10 & 11 & 5 & 43 & 21 \\
\hline Total & 91 & 10 & 50 & 16 & 16 & 10 & 11 & 5 & 209 & \\
\hline Percentage (\%) & 44 & 5 & 24 & 8 & 8 & 5 & 5 & 2 & & 100 \\
\hline
\end{tabular}




\section{PB rft patients}

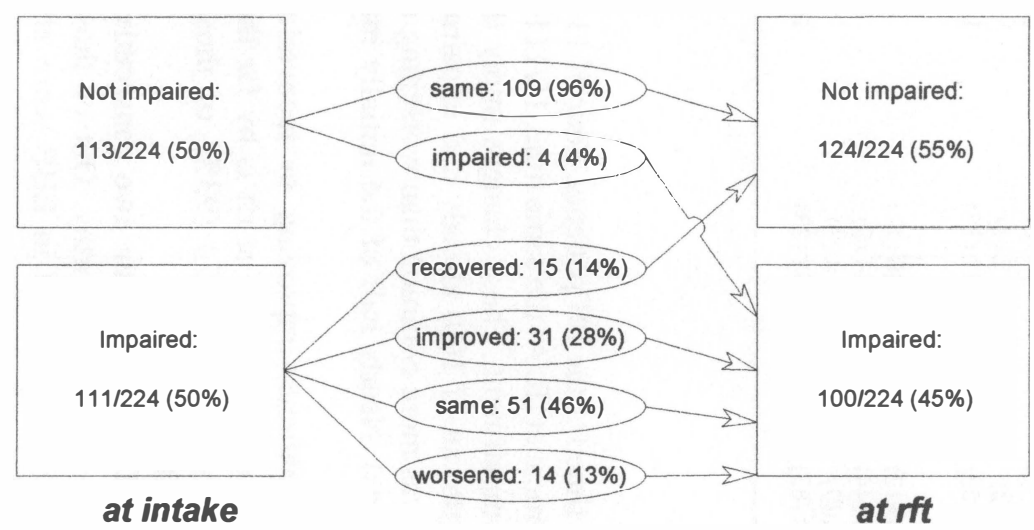

\section{PB follow-up patients}

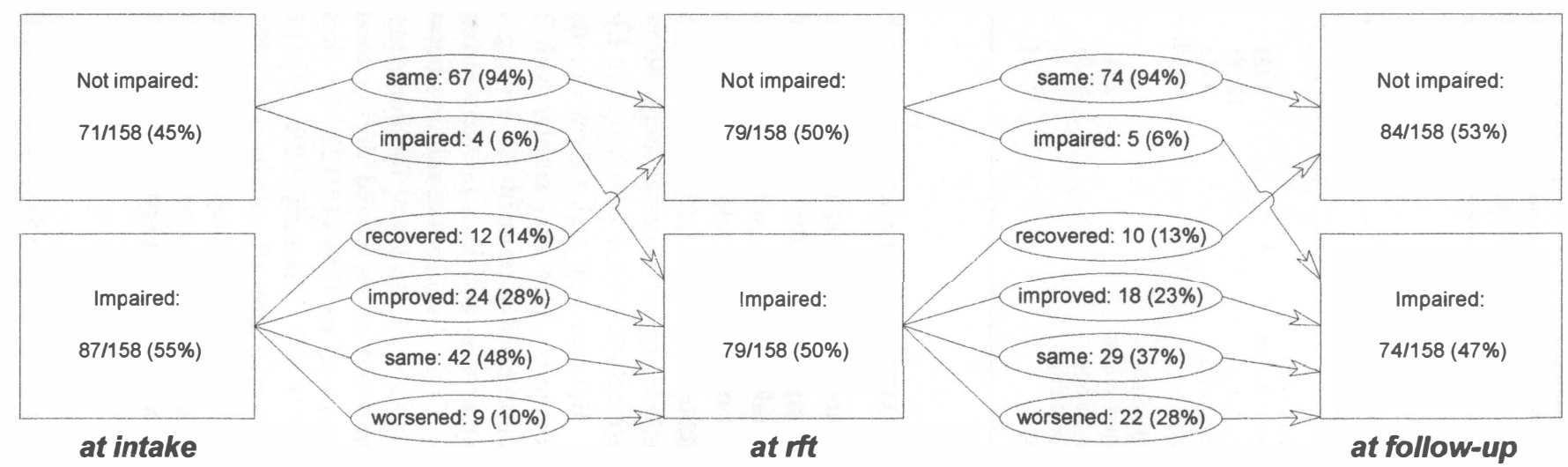


Table 2. Relation between changes in eye-hand-foot (EHF) score between intake and treatment completion ('intake $\rightarrow \mathrm{rft}$ ') and between treatment completion and latest assessment between 24 and 48 months after treatment completion ('rft $\rightarrow$ follow-up') for patients who completed treatment and were assessed for impairment grades at release from treatment and in the post-treatment period (percentages of all patients in brackets). In the table, 'improvement' refers to any gain and 'worsening' to any loss in EHF score

\begin{tabular}{|c|c|c|c|c|}
\hline \multirow[b]{2}{*}{$\begin{array}{l}\text { Change in EHF score } \\
\text { (intake } \rightarrow \mathrm{rft} \text { ) }\end{array}$} & \multicolumn{4}{|c|}{ Change in EHF score (rft $\rightarrow$ follow-up) } \\
\hline & $\begin{array}{c}\text { Improvement } \\
(\%)\end{array}$ & $\begin{array}{c}\text { No change } \\
(\%)\end{array}$ & $\begin{array}{c}\text { Worsening } \\
(\%)\end{array}$ & $\begin{array}{l}\text { Total } \\
(\%)\end{array}$ \\
\hline \multicolumn{5}{|l|}{ PB patients } \\
\hline Improvement & $8(5)$ & $16(10)$ & $12(8)$ & $36(23)$ \\
\hline No change & $12(8)$ & $84(53)$ & $13(8)$ & $109(69)$ \\
\hline Worsening & $8(5)$ & $3(2)$ & $2(1)$ & $13(8)$ \\
\hline Total & 28 (18) & $103(65)$ & $27(17)$ & $158(100)$ \\
\hline \multicolumn{5}{|l|}{ MB patients } \\
\hline Improvement & $2(1)$ & $20(12)$ & $20(12)$ & $42(25)$ \\
\hline No change & $10(6)$ & $72(44)$ & $14(8)$ & $96(58)$ \\
\hline Worsening & $11(7)$ & $12(7)$ & $4(2)$ & $27(16)$ \\
\hline Total & $23(14)$ & $104(63)$ & $38(23)$ & $165(100)$ \\
\hline
\end{tabular}

\section{DYNAMICS OF IMPAIRMENT IN PB PATIENTS}

Figure 1 summarizes changes in impairment over time for the PB patients. Four of $113(4 \%)$ patients free from impairment at intake had impairment at $\mathrm{rft}$. At the same time, 15/111 (14\%) initially impaired patients recovered from their impairment. These changes imply that the vast majority of patients with impairment at $\mathrm{rft}$ (96 out of 100) already had impairment at intake. Overall, improvement including recovery was more common than worsening (which includes onset of impairment, 46 versus 18 patients). Nearly half of the initially impaired patients $(46 \%)$ did not change in EHF score.

Between rft and follow-up, the numbers of patients improving or recovering and worsening were very similar with 28 and 27 . Having impairment at $\mathrm{rft}$ is by far the most important determinant for having impairment at follow-up: 69/74 (93\%) of those with impairment at follow-up were already impaired at $\mathrm{rtt}$.

Table 2 shows the relation between changes in EHF score in the two consecutive time intervals. A tendency for compensating changes is most noteworthy. Out of those who improved or recovered during treatment, 33\% (12/36) worsened in EHF score after rft, compared to $12 \%(15 / 122)$ for those who stayed the same or worsened during treatment $(P<0.005)$. Similarly, 62\% $(8 / 13)$ of patients who worsened during treatment improved or recovered after $\mathrm{rt}$, versus only $14 \%(20 / 145)$ of the others $(P<0 \cdot 001)$.

EHF score change does not necessarily reflect a patient's impairment dynamics well because improvement in one extremity or eye may coincide with worsening in another. Between intake and $\mathrm{rft}$, this actually happened in three patients (the EHF score did not change

Figure 1. Changes over time in impaiment status as measured by the eye-hand-foot (EHF) score for PB patients who completed treatment and whose impairment grades were assessed at release from treatment ('PB rft patients'), and for PB $\mathrm{rft}$ patients who in addition were assessed for impairment grades between 24 and 48 months after release from treatment ('PB follow-up patients'). 


\section{MB rft patients}

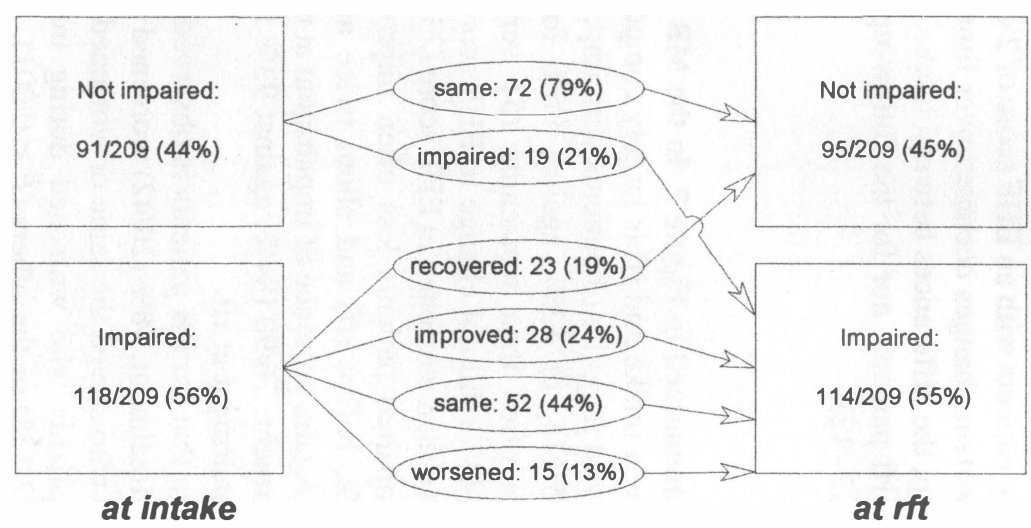

\section{MB follow-up patients}

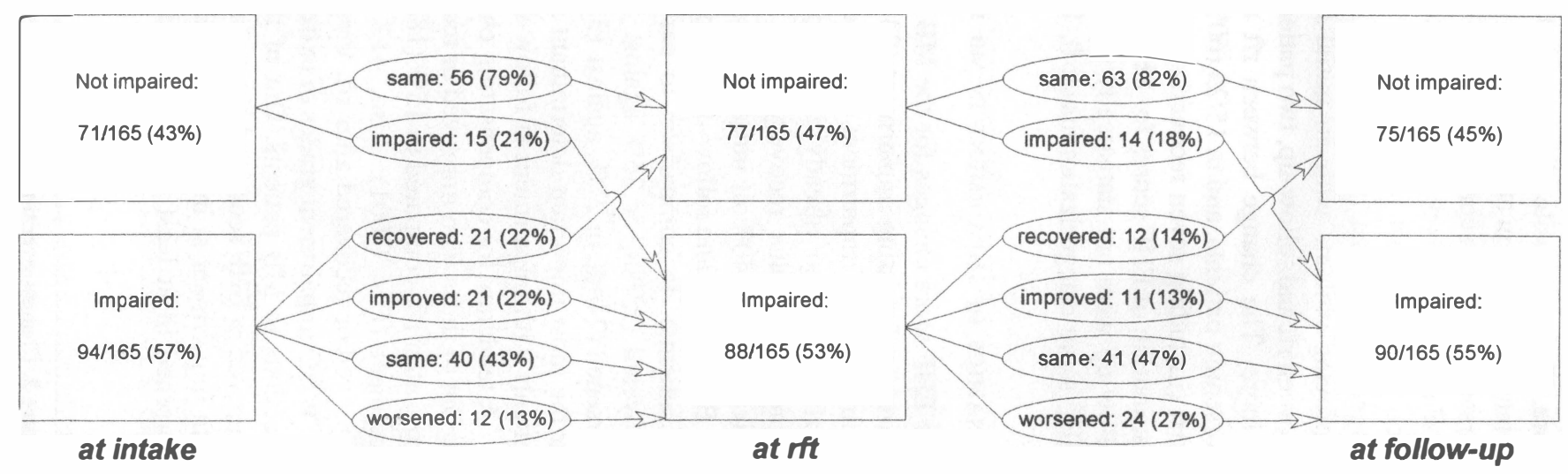


in one and improved in two of them). Further analysis revealed the same phenomenon in seven patients after rft. One of them worsened, two did not change and four improved in EHF score. These opposite changes imply that 35\% (28/79) of the already impaired follow-up patients worsened in at least one eye or extremity during follow-up, against $28 \%$ (22/79) who worsened in EHF score.

Further analysis revealed the maximum change in EHF score between intake and rft to be three points: one $\mathrm{rft}$ patient worsened, and two $\mathrm{rft}$ patients improved by three points. The change was at least two points in $33 \%$ of all worsening patients, and in $16 / 39(41 \%)$ of improving patients with an initial EHF score of 2 or more (including eight recoveries). Between rft and follow-up, two patients worsened, and four patients improved by three points or more. The change between rft and follow-up was at least two points in $41 \%$ of all worsening patients, and in 15/25 (60\%) of improving patients with an EHF score of 2 or more at rft (including seven recoveries). Overall, no important changes occurred over time in the distribution of EHF scores for PB patients as a group; the differences between intake, $\mathrm{rft}$ and follow-up were not statistically significant for the rft patients, nor for the follow-up group (EHF score categorization used: $0,1,2,3-4,5-6,7-12$ ).

\section{DYNAMICS OF IMPAIRMENT IN MB PATIENTS}

The EHF score changes for the MB patients are summarized in Figure 2. In the MB group, recovery of existing impairment ( 23 patients) between intake and $\mathrm{rft}$ is largely compensated by first onset of impairment (19 patients). Still, the vast majority of patients with impairment at $\mathrm{rft}$ were also already impaired at intake (95/114, or $83 \%$, against $96 \%$ for PB). Improvement plus recovery again occurred more often than worsening (51 versus 34 patients). Over $40 \%$ of initially impaired patients (44\%) did not change in EHF score.

Between rft and follow-up, less patients improved than worsened in EHF score (23 versus 38 patients). In comparison to earlier change, impaired patients less often improved or recovered $(23 / 88$, or $26 \%$ against $51 / 118$, or $43 \%$, before $\mathrm{rft}$ ) and about twice as of ten worsened $(24 / 88$, or $27 \%$, against $15 / 118$, or $13 \%)$. Again, presence of impairment at rft is by far the most important determinant for later impairment: 76/90 (84\%, against $93 \%$ for PB) patients with impairment at follow-up also had impairment at $\mathrm{rft}$.

A tendency for compensating changes similar to that for PB patients is observed (Table 2 ). Out of those who improved or recovered during treatment, $48 \%(20 / 42)$ worsened in EHF score after $\mathrm{rft}$, compared to $15 \%(18 / 123)$ for those who stayed the same or worsened during treatment $(P<0.001)$. Also, 41\% (11/27) of patients who worsened during treatment improved or recovered after $\mathrm{rft}$, versus only $9 \%(12 / 138)$ of the others $(P<0.001)$.

WHO impairment grades of extremities and eyes also simultaneously changed in opposite directions in $\mathrm{MB}$ patients. Out of the nine patients who experienced this before $\mathrm{rt}$, three improved in EHF score and six maintained their score. Because 13\% (15/118) of the patients with impairment at intake worsened in EHF score, this implies that 20\% (24/118) of them worsened in at least one eye, hand or foot. After $\mathrm{rft}$, only two MB patients experienced

Figure 2. Changes over time in impairment status as measured by the eye-hand-foot (EHF) score for MB patients who completed treatment and whose impairment grades were assessed at release from treatment ('MB rft patients'), and for MB rft patients who in addition were assessed for impairment grades between 24 and 48 months after release from treatment ('MB follow-up patients'). 
simultaneous improvement and worsening (one worsened and two of them did not change in EHF score).

Further analysis also demonstrated notable changes in the MB patients. Between intake and rft, six rft patients worsened, and eight rft patients improved by three EHF points or more. The total change was at least two points in $47 \%$ of all worsening patients, and in 33/47 (70\%) of improving patients with an initial EHF score of 2 or more (including 19 recoveries). Between rft and follow-up, six patients worsened, and one patient improved by 3 points or more. The total change between rft and follow-up was at least two points in $34 \%$ of all worsening patients, and in 10/20 (50\%) of improving patients with an EHF score of 2 or more at $\mathrm{rft}$ (including nine recoveries). Statistically significant differences in the EHF score distributions over time were not observed for the MB patients.

\section{Discussion}

The present study confirms the earlier indication that the AMFES cohort is severely affected by impairment and disability. ${ }^{7}$ Many patients were impaired at intake, frequently with WHO grade 2 and usually with multiple extremities involved. More than $10 \%$ of both PB and MB patients had EHF scores of 5 or more. Such scores imply very extensive nerve involvement.

DYNAMICS OF IMPAIRMENT OVER TIME

The dynamics of impairment over time were illustrated by comparing EHF scores between intake and $\mathrm{rft}$, and between $\mathrm{rft}$ and follow-up survey. Only a minority of patients with impairment at intake recovered completely. Impairment at the previous assessment was the most important determinant for impairment at the next. The dynamics of impairment were less favourable after $\mathrm{rft}$ than before. The risk of becoming impaired was both before and after rft significantly lower for PB than MB patients without previous impairment. During both periods, more than half of the impaired $\mathrm{PB}$ and $\mathrm{MB}$ patients changed in EHF score. A tendency towards compensation of EHF score improvement before rft by worsening after rft and vice versa was observed. Overall, the EHF score distributions of the PB and MB groups hardly changed over time.

Although the differences in the EHF score distributions at the different assessments were not statistically significant, the dynamics of impairment after rft deserve special attention. Compared with the treatment period, both $\mathrm{PB}$ and $\mathrm{MB}$ patients with impairment showed further worsening of their EHF score after rft twice as often. The EHF score measures both primary and secondary impairments. The development of primary impairments (sensory loss and muscle weakness) relates to active neuritis, which although it occurs, is much less common after rft than before. ${ }^{20}$ The worsening of the EHF score after rft is therefore likely to be due to increasing secondary impairment (wounds, ulcers and tissue loss), although the AMFES database does not contain this information in detail. This is in accordance with the suggestion from a study from Thailand that with longer periods after rft, changes in impairment status will more and more be due to new/increased tissue damage (e.g. wounds, bone loss) than to increases in NFI. ${ }^{5}$

Drop-out rates in our study were considerable. The greatest number of losses occurred at the time of the overthrowing of the former Ethiopian government in $1991 .{ }^{18}$ Probably, the longer duration of $\mathrm{MB}$ treatment contributes to the lower treatment completion rates in $\mathrm{MB}$ as 
compared to PB patients ( $71 \%$ versus $83 \%$ ). In contrast, follow-up of rft patients was more successful in the MB group (79\% examination versus $71 \%$ for PB). The drops-outs before and after rft differed from the other patients in several respects. Significant differences in EHF score change before rft were not observed between patients who did and did not drop out after rft. It must be noted that patients who experience complications may at times both be more prone (need for extra care) and less prone (due to loss of confidence in the programme, or hiding because of stigma) to complete treatment and to present at follow-up examinations.

Studies that address change in impairment over time $e^{3,5,8-10}$ are difficult to compare because of differences in case definitions, treatment durations and scoring systems for impairment. Still, all these studies found that clear majorities of patients with impairment at rft already had impairment at registration. One study also addressed change after $\mathrm{rft}^{5}$ In contrast to our study, the risks of worsening after rft were lower (but still significant) than before for MB patients and similar for PB patients. The EHF score was only utilized in two studies from Nepal. ${ }^{9,10}$ Both studies addressed the same group of MB patients at diagnosis and examination after two years of MDT. The percentage with impairment at diagnosis (44\%) was identical to our MB group. Although the percentage of patients with EHF scores of seven or more was higher in the Nepal group (6\% versus $2 \%$ ), the EHF score distributions at diagnosis were overall rather similar. Differences in the EHF score dynamics between the Nepal study and our study (usually rft was also 2 years later than intake) were observed, but a consistent pattern was not observed.

The dynamics of the EHF score after rft are worrisome. In addition, little is known about the long-term fate of leprosy patients who have irreversible nerve damage. The years of life lost to disability in this patient group, which accumulates over periods of many years, represents the real burden of leprosy disease. More insight into the size of this group, in the health related problems that they experience, in the care and support that they judge appropriate and in the associated resource requirements is urgently required. This patient group should get the attention in health policy agendas that it is entitled to.

\section{REFLECTION ON THE USE OF THE EHF SCORE}

We chose the EHF score as the evaluation tool for the present study. The EHF score gives a more detailed picture of the impairment status than the maximum WHO grade. In one of the two papers from Nepal, van Brakel et al. ${ }^{10}$ showed the EHF score to be much more sensitive than the maximum WHO impairment grade: $37 \%$ of patients who changed in EHF score did not change in maximum WHO grade. Further analysis showed this difference to be more pronounced in our study.

We agree with van Brakel that the EHF score is not a perfect impairment indicator: it remains a simple sum of the WHO impairment grades of the extremities and eyes. A point of criticism with respect to summary scores such as the EHF score is that they are unable to discriminate between a major change in one component and minor changes in several components. But for the WHO grades for extremities and eyes that make up the EHF score, van Brakel et al. ${ }^{10}$ stated that 'a change of one point at any site usually constitutes a major change in impairment status'. In extremities that improved in WHO impairment grade upon corticosteroid treatment, Broekhuis et al. ${ }^{16}$ showed the changes in sensory testing (ST) and voluntary muscle testing (VMT) to be important. Nevertheless, the EHF score may mask simultaneous changes of extremities and eyes in opposite directions. The frequency with which this happened in our study group is however not alarming. 
The reliability of the EHF score has not yet been established. To our knowledge, the retrospective study by Broekhuis et al. ${ }^{16}$ is the only study that investigated the reliability question. They indicated the hand-foot impairment score (sum of the WHO grades for extremities: HF score) to be a promising device for the evaluation of the effectiveness of corticosteroid treatment at programme level. They also demonstrated that the EHF score is not a suitable device for supporting individual patient management.

Unfortunately, it is not possible to validate the EHF score on the basis of the AMFES cohort. The main reasons for this are lack of detailed information on secondary impairment and the fact that the monitoring of AMFES patients was less close after $\mathrm{rft}$. Compared with other scoring systems, the EHF score has some important advantages. It is simple, reproducible, and information on its components (the WHO impairment grades for extremities) is already routinely collected in many control programmes. ${ }^{16}$ Although we acknowledged a number of deficiencies in the EHF score, we are convinced that they are outweighed by the practical usefulness of the EHF score. Following van Brakel ${ }^{10}$ and Broekhuis, ${ }^{16}$ we therefore strongly recommend initiation of prospective validation studies of the EHF score as tool for the evaluation of activities at programme level.

\section{Acknowledgements}

The dedicated work of the staff of the All Africa Leprosy, Tuberculosis and Rehabilitation Training Centre (ALERT) and financial support by the Netherlands Leprosy Relief (NLR) have made it possible to conduct this study and are gratefully acknowledged.

\section{References}

${ }^{1}$ Ponnighaus IM, Boerrigter G, Fine PE et al. Disabilities in leprosy patients ascertained in a total population survey in Karonga District, northern Malawi. Lepr Rev, 1990; 61: 366-374.

2 Schipper A, Lubbers WJ, Hogeweg M, De Soldenhoff R. Disabilities of hands, feet and eyes in newly diagnosed leprosy patients in eastern Nepal. Lepr Rev, 1994; 65: 239-247.

${ }^{3}$ Richardus JH, Finlay KM, Croft RP, Smith WC. Nerve function impairment in leprosy at diagnosis and at completion of MDT: a retrospective cohort study of 786 patients in Bangladesh. Lepr Rev, 1996; 67: 297-305.

${ }^{4}$ Ladhani S. Leprosy disabilities: the impact of multidrug therapy (MDT). Int J Dermatol, 1997; 36: 561-572.

5 Schreuder PA. The occurrence of reactions and impairments in leprosy: experience in the leprosy control program of three provinces in Northeastern Thailand, 1978-1995. III. Neural and other impairments. Int J Lepr Other Mycobact Dis, 1998; 66: 170-181.

6 Wittenhorst B, Vree ML, Ten Ham PB, Velema JP. The National Leprosy Control Programme of Zimbabwe: a data analysis, 1983-1992. Lepr Rev, 1998; 69: 46-56.

7 Meima A, Saunderson PR, Gebre S et al. Factors associated with impairments in new leprosy patients: the AMFES cohort. Lepr Rev, 1999; 70: 189-203.

8 Ponnighaus JM, Boerrigter G. Are 18 doses of WHO/MDT sufficient for multibacillary leprosy; results of a trial in Malawi. Int J Lepr Other Mycobact Dis, 1995; 63: 1-7.

9 Reed NK, van Brakel WH, Reed DS. Progress of impairment scores following commencement of chemotherapy in multibacillary leprosy patients. Int J Lepr Other Mycobact Dis, 1997; 65: 328-336.

10 van Brakel WH, Reed NK, Reed DS. Grading impairment in leprosy. Lepr Rev, 1999; 70: 180-188.

11 World Health Organization. International classification of impairments, disabilities and handicaps. World Health Organization, Geneva, 1980.

12 WHO Expert Committee on Leprosy. Sixth Report. WHO Technical Report Series No. 786, World Health Organization, Geneva, 1988.

13 WHO Expert Committee on Leprosy. Seventh Report. WHO Technical Report Series No. 874. World Health Organization, Geneva, 1998.

14 World-Health-Organization. Leprosy disabilities: magnitude of the problem. Wkly Epidemiol Rec, 1995; 70: 269-275. 
15 de Rijk AJ, Gabre S, Byass P, Berhanu T. Field evaluation of WHO-MDT of fixed duration at ALERT, Ethiopia: the AMFES project - I. MDT course completion, case-holding and another score for disability grading. Lepr Rev, 1994; 65: 305-319.

16 Broekhuis SM, Meima A, Koelewijn LF et al. The hand-foot impairment score as a tool for evaluating prevention of disability activities in leprosy: an exploration in patients treated with corticosteroids. Lepr Rev, 2000; 71: 344-354.

17 de Rijk AJ, Gabre S, Byass P, Berhanu T. Field evaluation of WHO-MDT of fixed duration, at ALERT, Ethiopia: the AMFES project - II. Reaction and neuritis during and after MDT in PB and MB leprosy patients. Lepr Rev, 1994; 65: 320-332.

18 Saunderson P, Gebre S, Desta K, Byass P. The ALERT MDT Field Evaluation Study (AMFES): a descriptive study of leprosy in Ethiopia. Patients, methods and baseline characteristics. Lepr Rev, 2000; 71: $273-284$.

19 Jopling WH. A practical classification of leprosy for field workers. Lepr Rev, 1981; 52: 273-277.

${ }^{20}$ Saunderson P, Gebre S, Desta K et al. The pattern of leprosy related neuropathy in the AMFES patients in Ethiopia: definitions, incidence, risk factors and outcome. Lepr Rev, 2000; 71: 285-308. 